\title{
Ocorrência de erros na administração de medicação - Revisão Integrativa
}

\section{Célia Sofia Barreiros Samico', Adriana Henriques², Pedro Lucas ${ }^{3}$}

\author{
${ }^{1}$ MScN, Nursing School of Lisbon. Lisbon, Portugal | email | https://orcid.org/0000-003- \\ 1587-0204 \\ ${ }^{2}$ PhD Nursing, Master Epidemiology, RN Coordinator Professor, Department \\ of Community Nursing, Researcher Nursing Research, Innovation and \\ Development Centre of Lisbon (CIDNUR). Nursing School of Lisbon and \\ Institute of Environmental Health, FMUL, Lisboa, Portugal | email | \\ https://orcid.org/0000-0003-0288-6653 \\ ${ }^{3} \mathrm{PhD}, \mathrm{MSc}, \mathrm{MScN}, \mathrm{RN}$, Nursing Research, Innovation and Development \\ Centre of Lisbon (CIDNUR). Nursing School of Lisbon. Lisbon, Portugal \\ prlucas@esel.pt | https://orcid.org/0000-0002-2560-7306
} outros tratamentos é um desafio para as organizações de saúde, e está muitas vezes na base da existência de eventos adversos. A administração de medicação é considerada uma atividade crucial no que concerne a segurança dos doentes. A ocorrência de erros durante 0 processo de gestão da medicação acarreta importantes custos para os doentes, profissionais e Serviço de Sáude. Nos países europeus 8 a 12\% dos doentes internados num hospital apresentaram eventos adversos sobretudo como erros de medicação. A Organização Mundial da Saúde definiu como terceiro Desafio Global de Segurança do Paciente, a Medicação sem danos. Objetivo: identificar a evidência científica sobre a ocorrência de erros na administração da medicação. Métodos: Revisão integrativa da literatura, com pesquisa nas bases de dados CINAHL, Cochrane, Mediclatina, MEDLINE, CUIDEN e referências bibliográficas dos artigos incluídos. Limitadores: artigos publicados de 2015 a 2020; texto integral e Europa. Resultados: Incluídos 9 artigos que descrevem relação entre ocorrência de erros e fatores da organização (Interrupções, recursos humanos insuficientes; ritmos trabalho acelerados, cargas de trabalho e cultura punitiva); fatores do sistema de medicação (sistemas de segurança) e fatores individuais (cansaço, falta de competências, negligência) Conclusão: Melhorar o ambiente da prática de enfermagem e garantir a existência de sistemas e rotinas de segurança na administração de medicação, uma cultura organizacional não punitiva e recursos humanos suficientes (treinados e com educação no uso seguro do medicamento), têm impacto na diminuição da ocorrência de erros de medicação e consequentemente, promotores da segurança do doente.

Palavras-chave: Segurança do doente; Erros de medicação; Enfermeiras.

Palavras-chave: Segurança do Doente; Erros de Medicação; Enfermeiras.

\section{Occurrence of Medication Administration Errors - Integrative Review}

Introduction: The increasing complexity of care associated with a greater use of new technologies, drugs and other treatments, proves to be a challenge for health organizations, and is often the basis for the existence of adverse events. The administration of medication is considered a crucial activity with regard to patient safety. The occurrence of errors during the medication management process entails important costs for patients, professionals and the health service. In regard to Europe, the data indicate that 8 to $12 \%$ of patients admitted to a hospital experienced adverse events, such as medication errors, among others. The World Health Organization has defined harmless medication as the third Global Patient Safety Challenge. Objective: To examine scientific evidence in medication administration errors Methods: Integrative literature review, based on database searches (CINAHL, Cochrane, Mediclatina, MEDLINE, CUIDEN) and bibliographic references of the articles included Limiters: articles published from 2015 to 2020; full text and Europe. Results: Nine included articles that report a relationship between the occurrence of errors and organizational factors (interruptions, lack of human resources, accelerated work rhythms, workloads and punitive culture); medication system factors (security systems) and individual factors (tiredness, lack of skills and negligence). Conclusion: Improve the nursing practice environment and ensure the existence of safety routines systems in the administration of medication, non-punitive organizational culture and sufficient human resources (trained and educated in the safe use of the medicine) have an impact on reducing the occurrence of medication errors and consequently, promoters of patient safety.

Keywords: Patient Safety; Medication Errors; Nurses. 


\section{Introdução}

Os erros de medicação são entendidos como um incidente evitável, decorrentes de má utilização do medicamento que podem levar a situações com impacto muito elevado nas pessoas e sistemas de saúde. Os erros de medicação estão associados a um custo anual, a nível mundial, de cerca de 42 biliões de dólares. Nos cuidados de saúde primários e em ambulatório, 4 em cada 10 doentes são prejudicados por algum evento e nos hospitais de países com economias de baixo ou médio rendimento, ocorrem 134 milhões de eventos adversos contribuindo para 2,6 milhões de mortes anuais (WHO, 2019). Na Europa os dados indicam que 8 a $12 \%$ dos doentes internados num hospital apresentaram eventos adversos, como erros de medicação. Dada a relevância foi definido como terceiro Desafio Global de Segurança do Doente a Medicação sem danos (WHO, 2017).

A segurança do doente, nas recomendações do Conselho da União Europeia integra quatro áreas de ação: políticas e programas; capacitação de doentes; notificação de eventos adversos e aprendizagem com os erros e educação e treino dos profissionais de saúde (WHO, 2017).

A World Health Organization (2019), define que para garantir melhorias sustentáveis e significativas ao nível da segurança do doente são necessárias: políticas claras, capacidade de liderança organizacional, profissionais de saúde qualificados, envolvimento efetivo dos doentes nos seus cuidados e dados que permitam promover melhorias na segurança.

Eventos adversos relacionados com o uso de medicamentos estão associados a sistemas de medicação fracos e ou fatores humanos como, fadiga, más condições ambientais ou falta de pessoal. (Alteren, Hermstad, White, \& Jordan, 2018; Harkanen, Blignaut, \& Vehvilainen-Julkunen, 2018b; Kim \& Kim, 2019; WHO, 2017).

Erros de medicação acontecem em várias etapas do ciclo de medicação, envolvendo diferentes profissionais. Os enfermeiros, responsáveis pela preparação e administração da medicação, têm um papel determinante em todo o processo, pelo que é necessário fundamental uma abordagem colaborativa evidenciando o seu o papel (Kavanagh, 2017).

O ambiente de prática de enfermagem (APE) é o fator mais influente e com maior impacto sobre os resultados de enfermagem e nas perceções da qualidade dos cuidados e da segurança do doente (Lucas \& Nunes, 2020). Melhorando este ambiente, tornando-o favorável, levará à melhoria dos resultados dos doentes e a um fator essencial para 0 aumento da satisfação dos enfermeiros (Leone et al., 2015, Shirey, 2006), fundamental para a manutenção de equipas com dotações seguras e nelas reter os enfermeiros (Lake, 2002).

Desenvolver APE favoráveis nos serviços de saúde é a melhor estratégia para garantir a segurança dos doentes (Almeida et al., 2020) e a qualidade dos cuidados de enfermagem (Aiken et al., 2012; Lucas \& Nunes, 2020; Wei et al., 2018; Wondmieneh, Alemu, Tadele, \& Demis, 2020; Zaghini et al., 2019).

A evidência científica aponta para uma lacuna em intervenções relacionadas com a educação dos enfermeiros no uso seguro da medicação (Morrudo et al., 2019; Millichamp \& Johnston, 2020; Wilson, Palmer, Levett-Jones, Giligan, \& Outram, 2016), identificando como barreiras aos relatos de erros na administração de medicação a cultura da organização, o sistema de notificação e comportamento da gestão. Destaca-se a necessidade de uma cultura de aprendizagem não punitiva e gestão de suporte para qualificar os enfermeiros para a gestão de erros (Vrbnjak et al., 2016).

O objetivo desta Revisão Integrativa da Literatura (RIL) é analisar a evidência científica acerca da ocorrência de erros na administração da medicação. 


\section{Material e Métodos}

Esta RIL integra as seguintes etapas: identificação da pergunta de pesquisa; definição das palavras-chave para pesquisa na literatura; definição das informações a serem extraídas dos estudos selecionados/categorização dos estudos; avaliação dos estudos incluídos na revisão integrativa; interpretação dos resultados; apresentação da revisão/síntese do conhecimento (Mendes, Silveira, \& Galvão, 2008; Whittemore \& Knafl, 2005).

A questão orientadora: Como se carateriza a ocorrência de erros na administração da medicação?

A pesquisa foi realizada nas bases de dados Cumulative Index to Nursing and Allied Health Literature (CINAHL) Complete, Cochrane Database of Systematic Reviews, Medical Literature Analysis and Retrievel System Online (MEDLINE) Complete e MedicLatina. Foi ainda consultada a base de dados CUIDEN.Foi igualmente explorada a literatura decorrente das citações dos artigos primários a partir das referências bibliográficas. A pesquisa foi realizada em julho de 2020, com os termos identificados nos Descritores em Ciências da Saúde (DeCS) e no Medical Subjet Heading (Mesh): patient safety; medication errors; nurses. O operador booleano usado foi AND. Foram aplicados como limitadores: artigos disponíveis em texto integral; data publicação: 2015-01-01/2020-12-31 e Europa.

$\mathrm{Na}$ inclusão dos estudos foi considerado: foco na ocorrência de erros relacionados com administração de medicação, com a intervenção de enfermeiros; disponíveis em texto integral e publicados em Inglês, Português ou Espanhol, na Europa, nos últimos cinco anos. Excluíram-se resumos de eventos científicos, estudos em que a metodologia não estava claramente definida. Foram identificados 82 artigos. Foram excluídos por leitura de título e duplicação em bases de dados, um total de 34 artigos. Dos 47 artigos restantes, foram excluídos 28 após leitura de resumo. Dos 19 remanescentes: 1 foi excluído sem texto integral; 11 por não responderem à questão ou aos critérios de inclusão. Nesta fase foram incluídos a partir de referências bibliográficas 2 artigos que responderam à pergunta de revisão. Na Figura 1 apresenta-se o processo de seleção Reporting ltems for Systematic Reviews and Meta-Analyses (PRISMA) de acordo com Moher, Liberati, Tetzlaff, Atman, \& The PRISMA Group (2009).

Para a inclusão dos 9 estudos procedeu-se à avaliação da qualidade metodológica dos artigos. Foram considerados os 7 níveis de evidência, agrupados segundo Melnyk \& Fineout-Overholt (2011), evidências fortes, moderadas e fracas. Foram respeitados os aspetos éticos da pesquisa no que concerne a autoria das ideias, assim como os conceitos e definições presentes nos estudos incluídos na RI. 


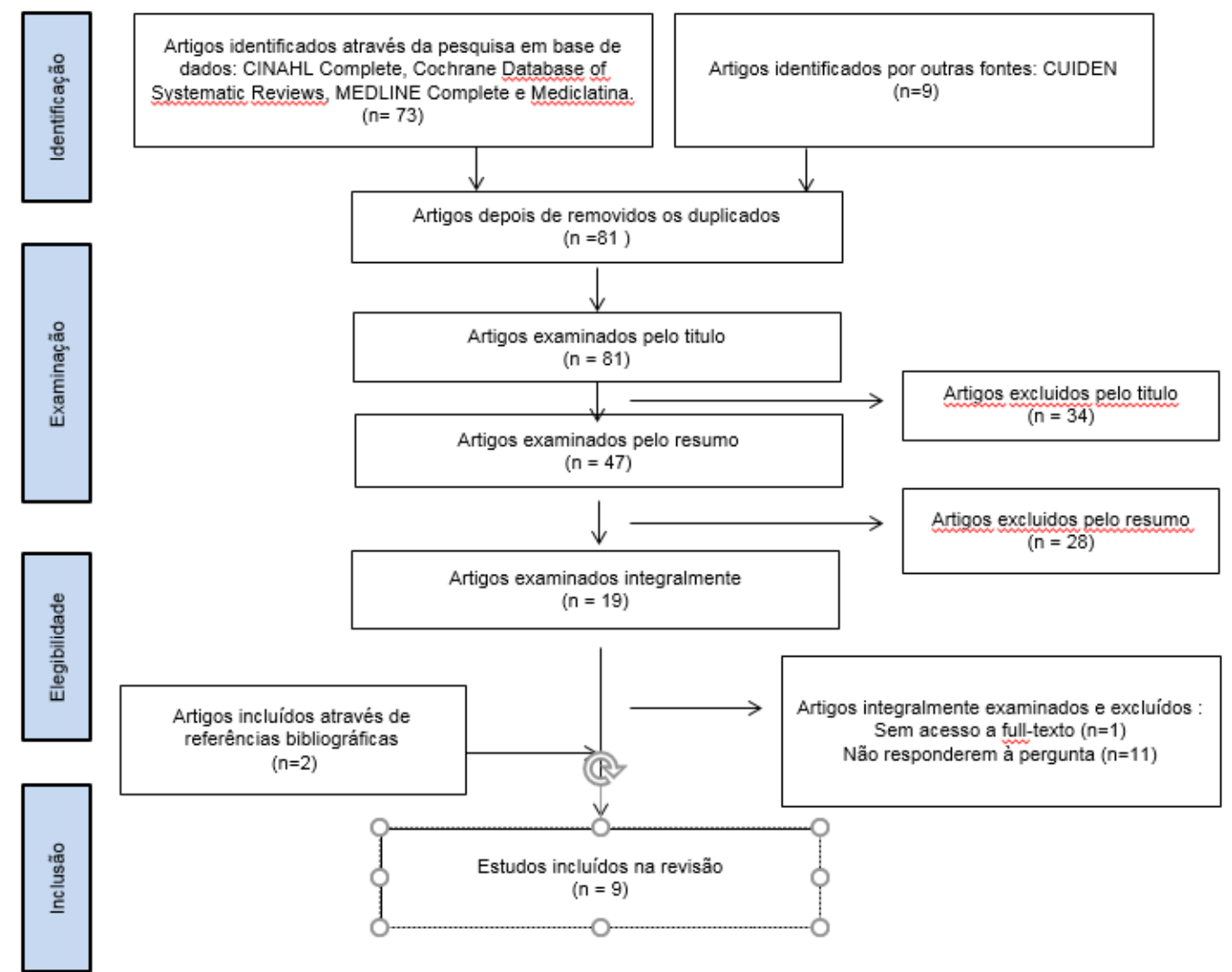

Fig. 1. Fluxograma do processo de identificação, seleção e inclusão dos estudos.

\section{Resultados}

Os 9 artigos forma analisados, quanto ao ano de publicação, um em 2015; um em 2016; um em 2017;

quatro em 2018 e dois em 2019. Todos foram publicados em revistas científicas e com pelo menos um autor do departamento de enfermagem, à exceção do estudo sueco em que o primeiro autor pertencia ao departamento de neurobiologia. Os estudos foram desenvolvidos, cinco (56\%) no Norte da Europa (Finlândia (2), Noruega (1), Suécia (1), e Reino Unido (1); $11 \%$ da Europa Central: República Checa (1) e três (33\%) no sudoeste da Europa: Itália (1), Turquia (1) e Sérvia (1). Oito (89\%) dos estudos apresentam um nível de evidência fraca (estudos descritivos e qualitativos) e um (11\%) moderada (estudo quase-experimental). Quanto à população alvo dos estudos, em seis $(67 \%)$ as amostras eram constituídas por enfermeiros, um (11\%) incluíam enfermeiros, farmacêuticos e médicos e em dois (22\%) estudos, as amostras eram doentes. Os estudos foram realizados em contexto hospitalar.

Os artigos analisados (síntese no Quadro 1) mencionam que a ocorrência de erros no processo de administração de medicação está relacionada com fatores como: interrupções durante administração de medicação (Alteren et al., 2018; Bower, Jackson, \& Manning, 2015; Mazzitelli, et al., 2018; Plutínská \& Plevová, 2019), recursos humanos insuficientes (Alteren et al., 2018; Harkanen et al.,2018a; Svitlica, Simin, \& Milutinovic, 2017), fatores individuais como cansaço, falta de habilidades ou negligência (Björkstèn et al., 2016; Härkänen, Tianen, \& Haatainen, 2018b); fatores relacionados com o sistema (Björkstèn et al., 2016; Edeer et al., 2019; Harkanen et al., 2018a; Plutínská \& Plevová, 2019), ambientes da prática de enfermagem (Alteren et al., 2018; Björkstèn et al., 2016; Harkanen et al., 2018a). 
Quadro 1. Síntese dos artigos incluídos na revisão

\begin{tabular}{|c|c|c|}
\hline $\begin{array}{l}\text { Estudos } \\
\text { Selecionados } \\
\text { Autores, Ano }\end{array}$ & Resultados & Conclusões \\
\hline $\begin{array}{l}\text { Alteren et al., } \\
(2018) .\end{array}$ & $\begin{array}{l}\text { Os enfermeiros são interrompidos durante a } \\
\text { administração de medicação, por motivos } \\
\text { evitáveis, principalmente por colegas e são } \\
\text { forçados a priorizar entre o atendimento ao } \\
\text { doente e a administração de medicamentos. }\end{array}$ & $\begin{array}{l}\text { Interrupções representam um risco } \\
\text { modificável e evitável para a administração } \\
\text { segura de medicamentos. A gestão deve } \\
\text { garantir que existem outros funcionários } \\
\text { disponíveis assegurar as necessidades dos } \\
\text { doentes }\end{array}$ \\
\hline $\begin{array}{l}\text { Björkstèn et al., } \\
(2016) .\end{array}$ & $\begin{array}{l}\text { Erros de medicação por negligência resultam da } \\
\text { interação complexa entre fatores individuais e } \\
\text { do sistema tal como os erros reportados no } \\
\text { resto do mundo. }\end{array}$ & $\begin{array}{l}\text { Os locais de trabalho devem trabalhar } \\
\text { sistematicamente com as rotinas de } \\
\text { segurança do doente e cuidar do bem-estar e } \\
\text { da educação contínua dos funcionários. }\end{array}$ \\
\hline $\begin{array}{l}\text { Bower et al., } \\
(2015) \text {. }\end{array}$ & $\begin{array}{l}\text { Interrupções têm um impacto negativo na } \\
\text { segurança do paciente. Esta revisão da } \\
\text { literatura identifica a natureza multidimensional } \\
\text { das interrupções e seu impacto na } \\
\text { administração de medicamentos e na } \\
\text { segurança do doente. }\end{array}$ & $\begin{array}{l}\text { É improvável que uma estratégia única sem } \\
\text { foco na segurança do doente seja capaz de } \\
\text { melhorar o efeito negativo das } \\
\text { interrupções. Necessária educação prática } \\
\text { para melhorar as interações da equipa e dotar } \\
\text { os enfermeiros com habilidades para gerir as } \\
\text { interrupções. }\end{array}$ \\
\hline $\begin{array}{l}\text { Edeer et al., } \\
(2019) .\end{array}$ & $\begin{array}{l}\text { O número médio de medicamentos utilizados } \\
\text { antes da admissão no hospital foi quatro. } 34,5 \% \\
\text { dos doentes apresentaram erros nos } \\
\text { medicamentos utilizados antes da admissão. } \\
\text { Nos medicamentos em que ocorreram erros } \\
25,2 \% \text { a } 32,2 \% \text { referem-se a antidiabéticos } \\
\text { orais, insulinas e medicamentos que atuam no } \\
\text { sistema cardiovascular. }\end{array}$ & $\begin{array}{l}\text { Erros de medicação resultaram de histórias } \\
\text { clínicas incompletas e imprecisas } \\
\text { relativamente aos medicamentos que os } \\
\text { doentes tomavam. Devem desenvolver-se } \\
\text { estratégias que forneçam informações } \\
\text { adequadas e precisas sobre os } \\
\text { medicamentos dos doentes usando registos } \\
\text { eletrónicos de dados. }\end{array}$ \\
\hline $\begin{array}{l}\text { Harkanen et al., } \\
(2018 a) .\end{array}$ & $\begin{array}{l}\text { Os desafios identificados quanto ao processo } \\
\text { de administração de medicação foram } \\
\text { agrupados em } 5 \text { categorias: medicamentos; } \\
\text { colaboração entre profissionais de saúde; } \\
\text { recursos e ambiente de trabalho; habilidades e } \\
\text { educação; e fatores relacionados com o doente. }\end{array}$ & $\begin{array}{l}\text { Para aumentar a segurança e a colaboração } \\
\text { eficaz, o sistema de saúde e a administração } \\
\text { do hospital devem manter os níveis de } \\
\text { pessoal necessários, recursos educacionais } \\
\text { contínuos e ambientes de trabalho calmos e } \\
\text { sem distrações. A indústria farmacêutica deve } \\
\text { facilitar o manuseamento de genéricos e } \\
\text { produtos LASA. }\end{array}$ \\
\hline $\begin{array}{l}\text { Härkänen et al., } \\
(2018 b) .\end{array}$ & $\begin{array}{l}\text { Incidentes relacionados com identificação } \\
\text { incorreta de doentes relacionaram-se com: } \\
\text { cansaço, falta de habilidades, negligência, } \\
\text { ritmos de trabalho rápidos e carga de trabalho } \\
\text { pesada. A importância de identificação correta } \\
\text { do doente para reduzir incidentes na } \\
\text { administração de medicação, pode não ser } \\
\text { completamente compreendida pelos } \\
\text { enfermeiros. }\end{array}$ & $\begin{array}{l}\text { Necessário maior suporte do sistema para } \\
\text { garantir a educação sobre a importância de } \\
\text { processos de identificação e de verificação de } \\
\text { medicação. Deve existir maior atenção aos } \\
\text { fatores organizacionais, como divisão do } \\
\text { trabalho, ritmos acelerados e carga de } \\
\text { trabalho. A comunicação verbal e escrita deve } \\
\text { ser mais eficaz. }\end{array}$ \\
\hline $\begin{array}{l}\text { Mazzitelli et al., } \\
(2018) \text {. }\end{array}$ & $\begin{array}{l}\text { Na coorte experimental as distrações dos } \\
\text { enfermeiros e conversas foram menores do que } \\
\text { no grupo controle. Distrações como: } \\
\text { telefonemas, outros doentes, ruído externo } \\
\text { resultaram maiores no grupo experimental. } \\
\text { Distrações relacionadas com: médicos, outros } \\
\text { funcionários, visitantes e emergências não } \\
\text { apresentaram diferenças significativas. }\end{array}$ & $\begin{array}{l}\text { Usar colete de alta visibilidade durante a } \\
\text { administração de drogas provou ser muito útil. } \\
\text { É importante adotar sinais de "não perturbe" e } \\
\text { programas educacionais dirigidos a toda a } \\
\text { equipa. }\end{array}$ \\
\hline $\begin{array}{l}\text { Plutínská \& } \\
\text { Plevová, (2019). }\end{array}$ & $\begin{array}{l}\text { Estudos avaliaram intervenções como: } \\
\text { envolvimento farmacêutico, dispositivos de } \\
\text { infusão automatizada, relato de erros de } \\
\text { medicação, estratégias para limitar interrupções } \\
\text { durante a administração de medicamentos, } \\
\text { registos eletrônicos de saúde, assim como } \\
\text { sistemas de apoio à tomada de decisão clínica, } \\
\text { educação e criação de listas de verificação } \\
\text { relativas à administração de medicamentos. }\end{array}$ & $\begin{array}{l}\text { Todos os estudos apresentaram certa } \\
\text { redução no erro de medicação. } \\
\text { Devido a inúmeras limitações, no } \\
\text { entanto, é impossível selecionar e } \\
\text { recomendar uma única abordagem. }\end{array}$ \\
\hline $\begin{array}{l}\text { Svitlica et al., } \\
(2017) \text {. }\end{array}$ & $\begin{array}{l}\text { O fator dominante para a ocorrência de erros foi } \\
\text { o número insuficiente de enfermeiros. Outros } \\
\text { fatores dominantes apresentados na literatura } \\
\text { não foram reconhecidos neste estudo. }\end{array}$ & $\begin{array}{l}\text { As instituições de saúde devem promover a } \\
\text { educação sobre o uso seguro de medicação e } \\
\text { programas de educação continua. É } \\
\text { importante que os enfermeiros reconheçam o } \\
\text { que é um erro, as causas e a importância de } \\
\text { os relatar e analisar. }\end{array}$ \\
\hline
\end{tabular}




\section{Discusão}

Os estudos de ocorrência de erros na administração de medicação, ao nível Europeu, são maioritariamente estudos descritivos e qualitativos de evidência fraca (Melnyk \& FineoutOverholt, 2011), indicando a necessidade de realização de investigação clínica de forte evidência.

Os fatores relacionados com a ocorrência de erros forma classificados em: fatores do sistema de medicação, fatores do APE e organização e fatores humanos.

\subsection{Fatores do Sistema de Medicação}

Identifica a ocorrência de erros associados a registos incompletos ou imprecisos, omissão de medicamentos nas histórias clínicas dos doentes; necessidade de formação no manuseamento de produtos genéricos e Look-alike sound-alike (LASA), identificação incorreta de doentes e ausência de notificação de erros de medicação (Björkstèn et al., 2016; Edeer et al., 2019; Harkanen et al., 2018 a; Harkanen et al., 2018 b). O estudo de Plutínská \& Plevová, (2019), indica que registos eletrónicos de saúde, sistemas de apoio à tomada de decisão e criação de listas de verificação de medicação apresentam redução no erro.

\subsection{Fatores do APE e Organização}

Fatores relacionados com o ambiente da prática de enfermagem, são os mais mencionados. Sete (64\%) estudos referem-se a interrupções (Alteren et al., 2018; Bower et al., 2015; Mazzitelli et al., 2018; Plutínská \& Plevová, 2019), a recursos humanos insuficientes (Alteren et al., 2018; Harkanen et al., 2018a; Svitlica et al., 2017), a ritmos de trabalho rápidos e cargas de trabalho pesadas (Härkänen et al., 2018b).

Para o uso seguro de medicação, a necessidade e relevância atribuída a programas de educação contínua dirigida a enfermeiros, é apontada em $73 \%$ dos estudos analisados (Björkstèn et al., 2016; Bower et al., 2015; Harkanen et al., 2018a; Härkänen et al., 2018b; Mazzitelli, et al., 2018; Plutínská \& Plevová, 2019; Svitlica et al., 2017).

Nos estudos analisados é determinante o papel da gestão para promover recursos humanos, ritmos, cargas de trabalho adequados (Alteren et al., 2018; Harkanen et al., 2018a; Härkänen et al., 2018b; Svitlica et al., 2017); assegurar a existência de rotinas e sistemas de segurança no uso de medicamentos (Björkstèn, et al., 2016; Edeer et al., 2019; Plutínská \& Plevová, 2019); promover uma cultura de aprendizagem não punitiva (Svitlica et al., 2017) e implementar programas de educação sobre o uso seguro da medicação (Björkstèn et al., 2016; Bower et al., 2015; Harkanen et al., 2018a; Härkänen et al., 2018b; Mazzitelli, et al., 2018; Plutínská \& Plevová, 2019; Svitlica et al., 2017).

\subsection{Fatores Humanos}

Os fatores humanos surgem associado a fatores do sistema que podem resultar em erros por negligência (Björkstèn et al., 2016). As barreiras pessoais e profissionais como medo, responsabilidade e caracteristicas dos enfermeiros que levam à omissão dos erros. Härkänen et al., (2018b) menciona o cansaço e a falta de habilidades como fatores que levaram a incidentes de administração de medicação a doentes errados.

A análise realizada permitiu identificar a natureza multidimensional da ocorrência de erros na administração de medicação, indo de encontro à evidência científica disponível existente. Revela-se fulcral compreender e investigar este fenómeno para implementar medidas que o tornem seguro, contribuindo para a melhoria contínua dos cuidados $e$ segurança dos doentes. 


\subsection{Limitações da RIL}

Utilização de limitadores na pesquisa: textos completos e estudo Europa pode ter levado à omissão de trabalhos relevantes. $O$ fato da maioria dos estudos apresentarem evidência fraca também se constitui como uma limitação.

\subsection{Implicações para a Prática}

Para evitar a ocorrência de erros de medicação, contribuindo para a segurança do doente é determinante que as organizações adotem uma cultura de aprendizagem não punitiva. É importante que entre a gestão e os enfermeiros se desenvolvam políticas de comunicação abertas e de suporte. A gestão deve assegurar dotações seguras de enfermagem nos serviços e promover educação contínua no âmbito do uso seguro da medicação, como estratégias para garantir a administração segura de medicação. Observa-se escassez de pesquisa de evidência forte, acerca da ocorrência de erros na medicação, na Europa. Esta revisão permitiu identificar a complexidade subjacente a eventos adversos relacionados com o uso de medicação, desempenhando um importante papel no que concerne ao desenvolvimento de práticas baseadas na evidência. Seria importante desenvolver pesquisa no âmbito de intervenções, como programas educativos e implementação de sistemas de medicação que permitam perceber os resultados na segurança dos doentes.

\section{Conclusões}

Esta RIL permitiu sintetizar evidência relacionada com a ocorrência de erros na administração de medicação. A maioria dos estudos são qualitativos ou descritivos e maioritariamente dirigidos a enfermeiros em contexto hospitalar. O estudo da ocorrência de erros na administração de medicação, a partir da metodologia qualitativa com recurso a diversas fontes de dados permitiu uma compreensão sistematizada mais abrangente e holística do problema. Subjacente à ocorrência de erros identificaram-se os seguintes fatores: fatores do sistema de medicação, fatores organizacionais e fatores individuais.

Para reduzir a ocorrência de erros na administração de medicação, por parte dos enfermeiros é fulcral investir em programas de educação contínua sobre o uso seguro da medicação e adotar uma cultura organizacional não punitiva. A gestão assume um papel primordial competindo-Ihe garantir: existência de recursos humanos adequados para evitar cargas de trabalho excessivas e ritmos de trabalho acelerados; promover ambientes de prática de enfermagem favoráveis sem interrupções e implementar sistemas de segurança no uso da medicação.

\section{Referências}

Aiken, L., Sermeus, W., Van den Heed, K., Sloane, D., Buss, R., McKee, M., \& Bruyneel, L. (2012) Patient safety, satisfaction, and quality of hospital care:cross sectional surveys of nurses and patients in 12 countries in Europe and the United States. British Medical Journal, 1-14. doi:10.1136/bmj.e1717.

Almeida, S., Nascimento, A., Lucas, P. B., Jesus, E. \& Araújo, B. (2020). RN4CAST Study in Portugal:

Validation of the Portuguese Version of the Practice Environment Scale of the Nursing Work Index. Aquichan, 20(3), e2038. doi:10.5294/aqui.2020.20.3.8.

Alteren, J., Hermstad, M., White, J., \& Jordan, S. (2018). Conflicting priorities: Observation of medicine administration. Journal of Clinical Nursing, 3613-3621. doi:10.1111/jocn.14518.

Björkstèn, K., Bergqvist, M., Andersèn-Karlsson, E., Benson, L., \& Ulfvarson, J. (2016). Medication errors as malpractice-a qualitative content analysis of 585 medication errors by nurses in Sweden. BMC Health Services Research, 16(1). doi:10.1186/s12913-016-1695-9. 
Bower, R., Jackson, C., \& Manning, J. (2015). Interruptions and medication administration in critical care. Nursing in Critical Care, 20(4). doi:10.1111/nicc.12185.

Clendon, J., \& Gibbons, V. (2015). $12 \mathrm{~h}$ shifts and rates of error among nurses: A systematic review. International Journal of Nursing Studies, 52(7), 1231-1242. doi:10.1016/j.jnurstu.2015.03.011.

Edeer, A., Serap, K., Evsine, N., Cengis, G., \& Apaydin, F. (2019). Medication Errors Related to the Clinical Use of Medications Used by Cardiovascular Surgery Patients before Admission to Hospital: A Cross-Sectional Study. International Journal of Caring Sciences, 12(3), 13321339.

Eltaybani, S., Abdelwareth, M., Abou, Z. N., \& Ahmed, N. (2020). Recommendations to prevent nursing errors: Content analysis of semi-structured interviews with intensive care unit nurses in a developing country. Journal of Nursing Management, 28(3), 690-698. doi:10.1111/jonm.12985.

Harkanen, M., Blignaut, A., \& Vehvilainen-Julkunen, K. (2018a). Focus group discussions of registered nurses perceptions of challenges in the medication administration process. Nursing \& Health Sciences, 1-7. doi:10.1111/nhs.12432.

Härkänen, M., Tianen, M., \& Haatainen, K. (2018b). Wrong-patient incidents during medication administrations. Journal of Clinical Nursing, 27(3-4), 715-724. doi:10.1111 / jocn.14021.

Lake, E. T. (2002). Development of the practice environment scale of the nursing work index. Research Nursing Health. 25(3):176-88. doi: 10.1002/nur.10032.

Leone, C., Bruyneel, L., Anderson, J. E., Murrells, T., Dussault, G., Jesus, É. H. et al. (2015). Work environment issues and intention-to-leave in Portuguese nurses: a cross-sectional study. Health Policy. 119(12):1584-92. doi: 10.1016/j.healthpol.2015.09.006.

Lucas, P. R. M. B., \& Nunes, E. M. G. T. (2020). Nursing practice environment in Primary Health Care: a scoping review. Revista Brasileira de Enfermagem, 73(6), e20190479. doi:10.1590/0034-7167-2019-0479.

Kavanagh, C. (2017). Medication governance: preventing errors and promoting patient safety. Br J Nurs, 26(3):159-165. doi:10.12968/bjon.2017.26.3.159

Kim, M., \& Kim, C.-H. (2019). Canonical correlations between individual self-efficacy/organizational bottom-up approach and perceived barriers to reporting medication errors: a multicenter study. BMC Health Services Research, 19(495). doi:10.1186/s12913-019-4194-y.

Mazzitelli, N., Rocco, G., De Andreis, G., Mauro, L., Montevecchi, A., Stievano, A., \& Turci, C. (2018). Reducing drug administration errors using "Do not disturb" tabards and signs. Professioni infermieristiche, 71(2), 95-103. http://www.profinf.net/pro3/index.php/IN/article/view/499.

Melnyk, B., \& Fineout-Overholt, E. (2011). Evidence-based practice in nursing \& healthcare: $A$ guide to best practice. Philadelphia: Lippincott Williams \& Wilkins.

Mendes, K. D., Silveira, R. C., \& Galvão, C. M. (2008). Revisão Integrativa: Método de pesquisa para a incorporação de evidências na saúde e na enfermagem. Texto Contexto Enfermagem, 758-764. Retrieved from http://www.scielo.br/pdf/tce/v17n4/18.pdf.

Millichamp, T., \& Johnston, A. (2020). Interventions to support safe medication administration by emergency department nurses: An integrative review. International Emergency Nursing, 49. doi:10.1016/j.ienj.2019.100811.

Moher, D., Liberati, A., Tetzlaff, J., Atman, D., \& The PRISMA Group . (2009). Preferred reporting items for systematic reviews and meta-analyses: the PRISMA statement. PloS medicine, 6 (7), e1000097. doi:10.1371/journal.pmed.1000097.

Morrudo, E., Figueiredo, E., Silveira, R., Barlem, J., Oliveira, S., \& Ramos , F. (2019). Errors in medicinal therapy and the consequences for nursing. Revista de Pesquisa: Cuidado $e$ Fundamental, 11(1), 88-96. doi:10.9789/2175-5361.2019.v11i1.88-96.

Plutínská, Z., \& Plevová, I. (2019). Measures to Prevent Medication Errors in Intensive Care Units. Central European Journal of Nursing \& Midwifery, 10(2), 1059-1067. doi:10.15452/CEJNM.2019.10.0014. 
Poghosyan, L., Shang, J., Poghosyan, H., Liu, N., \& Berkowitz, B. (2015). Nurse practitioners as primary care providers: Creating favorable practice environments in New York State and Massachusetts. Health Care Management Review, 40(1), pp. 46-55. doi:10.1097/HMR.0000000000000010.

Shirey, M. R. (2006). Authentic Leaders Creating Healthy Work Environments for Nursing Practice. American Journal of Critical Care. 15(3):256-67. http://ajcc.aacnjournals.org/ content/15/3/256.full.pdf+html.

Svitlica, B., Simin, D., \& Milutinovic, D. (2017). Potential causes of medication errors: perceptions of Serbian nurses. 64(3), 421-427. International Nursing Review, 64(3), 421-427. doi:10.1111/inr.12355

Vrbnjak, D., Denieffe, S., Gorman, C., \& Pajnkihar, M. (2016). Barriers to reporting medication errors and near misses among nurses. International Journal of Nursing Studies, 63, 162-178. doi:10.1016/j.ijnurstu.2016.08.019.

Wei, H., Sewell, A. K., Woody, G., \& Rose, A. M. (2018). The state of the science of nurse work environments in the United States: A systematic review. Journal of Nursing Studies, 5(3), 287-300. doi:10.1016/j.ijnss.2018.04.010.

Whittemore, R. \& Knafl, K. (2005). The integrative review: updated methodology. Journal of Advanced Nursing, 52(5), 546-553. doi:10.1111/j.1365-2648.2005.03621.x.

Wilson, A., Palmer, L., Levett-Jones, T., Giligan, C., \& Outram, S. (2016). Interprofessional collaborative practice for medication safety: Nursing, pharmacy, and medical graduates' experiences and perspectives. Journal of Interprofessional Care, 30(5), 649-654. doi:10.1080/13561820.2016.1191450.

Wondmieneh, A., Alemu, W., Tadele, N., \& Demis, A. (2020). Medication administration errors and contributing factors among nurses: a cross sectional study in tertiary hospitals, Addis Ababa, Ethiopia. BMC Nursing, 19(4). doi:doi.org/10.1186/s12912-020-0397-0

World Health Organization (2017). Medication Without Harm - Global Patient Safety Challenge on Medication Safety. Geneva, Switzerland. https://www.who.int/patientsafety/medicationsafety/medication-without-harm-brochure/en/

World Health Organization (2019). Medication Safety in Transitions of Care. https://www.who.int/patientsafety/en/

Zaghini, F., Fiorini, J., Piredda, M., Fida, R., \& Sili, A. (2019). The relationship between nurse managers' leadership style and patients' perception of the quality of the care provided by nurses: Cross sectional survey. International Journal of Nursing Studies, 101, 103446. doi:10.1016/j.jinurstu.2019.103446. 